\title{
Adjoint-based sensitivity analysis of ignition in a turbulent reactive shear layer
}

\author{
Jesse Capecelatro* \\ University of Michigan, Ann Arbor, Michigan, 48109-2125, USA \\ Daniel J. Bodony ${ }^{\dagger}$ and Jonathan B. Freund ${ }^{\ddagger}$ \\ University of Illinois at Urbana-Champaign, Urbana, Illinois, 61801, USA
}

\begin{abstract}
The uncertainty of successful ignition in non-premixed turbulent flows remains a fundamental challenge in many practical combustion systems. Mapping the spatial distribution of ignition probability often relies on iterative testing that becomes prohibitive when measuring sensitivity to a large number of parameters or varied flow conditions. An accelerated approach is to formulate the adjoint of the perturbed and linearized governing equations in such a way that sensitivity of an ignition indictor to an arbitrarily large number of parameters can be obtained with a cost comparable to the flow solution. The purpose of the present study is to demonstrate the capability of an adjoint solution to inform parametric uncertainty and optimal ignition placement in turbulent non-premixed combustion. Direct numerical simulations of assisted ignition in a non-premixed hydrogen-air mixing layer are presented. Adjoint-based sensitivity of ignition likelihood to placement of the igniter are reported for different flow realizations.
\end{abstract}

\section{Introduction}

The reliable and accurate control of ignition in fuel-air mixtures has remained a key challenge in the design of many practical combustion systems. Many proposed supersonic flight vehicles operate in the autoignition mode, where the temperature at the inlet of the combustion chamber causes spontaneous ignition downstream where fuel and oxidizer mix. Spontaneous ignition of hydrogen fuel requires conditions prohibitive for hypersonic flights and increases the probability of unstart. ${ }^{1-3}$ Furthermore, autoignition relies on fuel-rich environments that contribute to harmful $\mathrm{NO}_{x}$ emissions. Introducing an external energy source, such as a laser spark or an electric arc, can be used to ignite the flow in conditions where autoignition does not occur. Placing the igniter upstream of the fuel injection site was recently observed to increase the fuel-mixing length, enhance the air-fuel mixing due to baroclinic torque effects, and improve combustion performance downstream via radical farming. ${ }^{3}$

A key challenge in designing practical combustors with external ignitors is determining the minimum energy required for successful ignition (MEI). ${ }^{4-6}$ MEI has been shown to be strongly dependent on the ignition parameters, ${ }^{5}$ with flow inhomogeneities (due to turbulence for example) increasing MEI. ${ }^{6}$ In general, the initiation and propagation of a flame from a cold (non-reacting) mixture is sensitive to the instantaneous flow conditions, local mixture fraction, and position, extent, and intensity of the ignition source. ${ }^{7,8}$ Numerous studies can be found in the literature seeking to better understand assisted ignition in turbulent flows. However, these studies often rely on trial-and-error testing. ${ }^{9-13}$

The sensitivity of ignition to a set of control parameters provides a means to adjust these parameters to seek MEI. ${ }^{14}$ The objective of the present study is to assess the sensitivity of ignition in turbulent nonpremixed combustion by performing direct numerical simulation and computing its corresponding space-time discrete-adjoint. The solution to the adjoint equations is particularly attractive because it provides sensitivity

*Assistant Professor. University of Michigan, Department of Mechanical Engineering. AIAA Member.

${ }^{\dagger}$ Associate Professor. University of Illinois, Aerospace Engineering. AIAA Associate Fellow.

¥Professor. University of Illinois, Mechanical Science \& Engineering and Aerospace Engineering. AIAA Associate Fellow. 


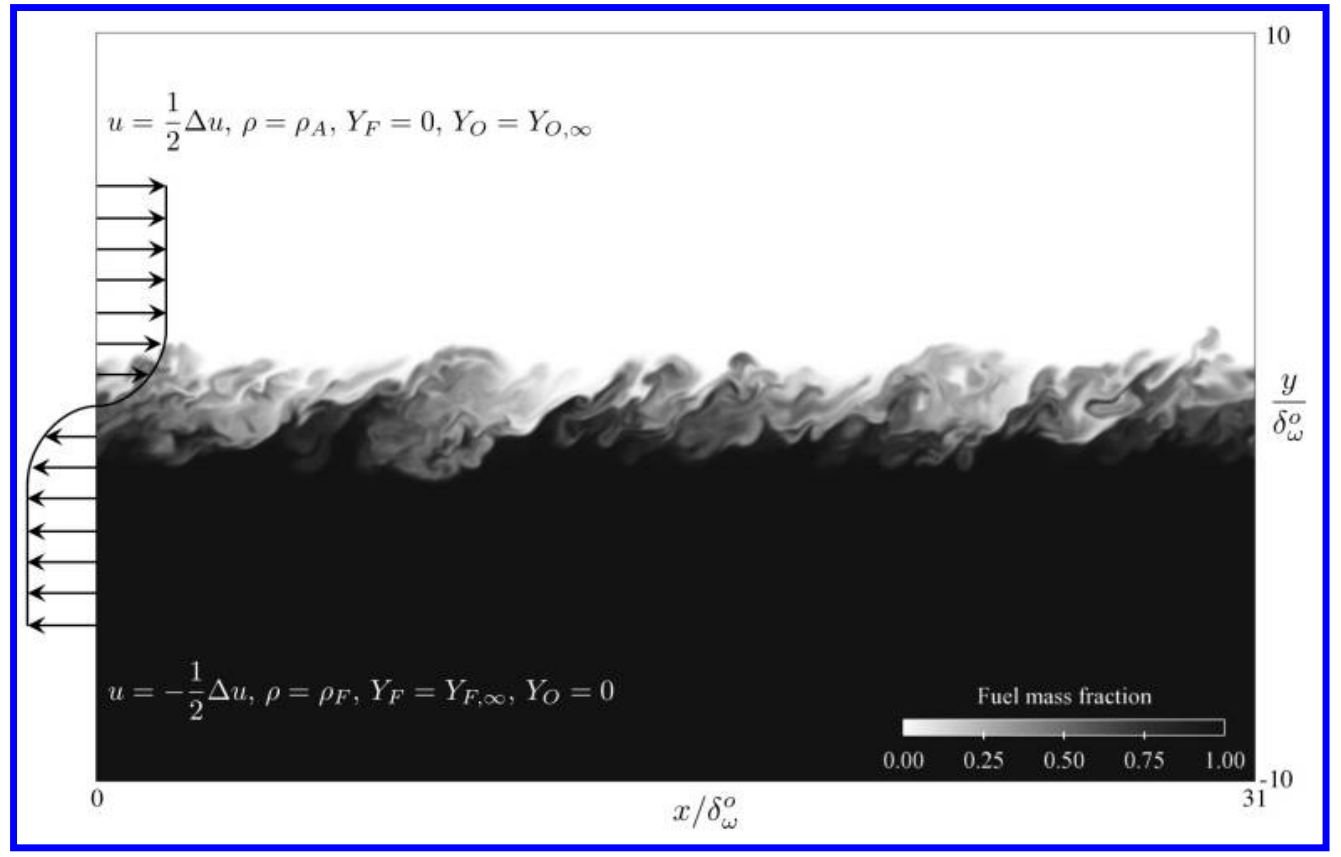

Figure 1. Visualization of the temporally developing hydrogen-air mixing layer showing fuel mass fraction (black/white), scaled with initial vorticity thickness $\delta_{\omega}^{o}$.

of a quantity of interest with a cost that is comparable to the flow solution. A discrete-adjoint method is used to provide a sensitivity gradient that is exact up to floating-point induced roundoff errors. To isolate the effects of turbulence on non-premixed combustion, we simulate ignition in the time-developing turbulent hydrogen-air mixing layer shown in Fig. 1. Ignition is initiated by depositing energy at a specified location in the flow. Sensitivity of ignition on the vertical and horizontal placement in the shear layer is computed for a range of flow realizations. The relative importance of each parameter used in defining the external igniter is then assessed, and its variation at different locations is discussed.

\section{Flow configuration}

The computational setup involves two streams of air and hydrogen flowing in opposite directions, as depicted in Fig. 1. The upper stream consists of fuel with mass fraction $Y_{F \infty}=0.1$ and velocity $\Delta u / 2$, and lower stream of oxidizer with mass fraction $Y_{O \infty}=0.233$ and velocity $-\Delta u / 2$. The initial Reynolds number based on $\Delta u$ and the initial vorticity thickness, $\delta_{\omega}^{o}$, is $R e_{\delta_{\omega}^{o}}=2600$, where

$$
\delta_{\omega}^{o}=\frac{\Delta u}{|d \tilde{u} / d y|_{\max }},
$$

with $\tilde{u}$ the Favre-average streamwise velocity and $y$ the vertical coordinate. The convective Mach number for the two streams is defined as $M_{c}=\Delta u /\left(c_{A}+c_{F}\right)=0.3$, where $c_{A}$ and $c_{F}$ denote the speed of sound in the air and fuel stream, respectively. This value is small enough such that compressibility effects from are not important. The density is taken to be uniform across the two streams to eliminate the effects of density differences on turbulence. As a result, the air stream has an initial temperature approximately $20 \%$ higher than the fuel side, but the temperature is low enough such that auto-ignition does not occur. The mixing layer is simulated on a Cartesian grid of $1484 \times 513 \times 384$ points in $x, y$, and $z$, respectively, with side lengths $L_{x}=31 \delta_{\omega}^{o}, L_{y}=20 \delta_{\omega}^{o}$, and $L_{z}=8 \delta_{\omega}^{o}$. This flow configuration is similar to that of Pantano et al. ${ }^{15}$ but with approximately twice the resolution in order to properly resolve the flame thickness for the chemical kinetics described in the following section. Grid stretching applied in the $y$-direction varies smoothly from $\Delta y=0.9 \Delta x$ at the centerline to $\Delta y=6 \Delta x$ at the boundary. After an initial transient, quasi-linear growth is observed in $\delta_{\omega}$ and the momentum thickness, $\delta_{\theta}$, as shown in Fig. 2. Here, the momentum thickness is 
defined as

$$
\delta_{\theta}=\frac{1}{\rho_{\infty} \Delta u^{2}} \int_{-\infty}^{\infty} \bar{\rho}\left(\frac{1}{4} \Delta u^{2}-\widetilde{u}^{2}\right) d y
$$

where $\rho_{\infty}$ is the free stream density of the air and $\bar{\rho}$ is the mean density at $y$. At this point in time, thermal energy is deposited to a local region of the flow to seed ignition of the mixture.

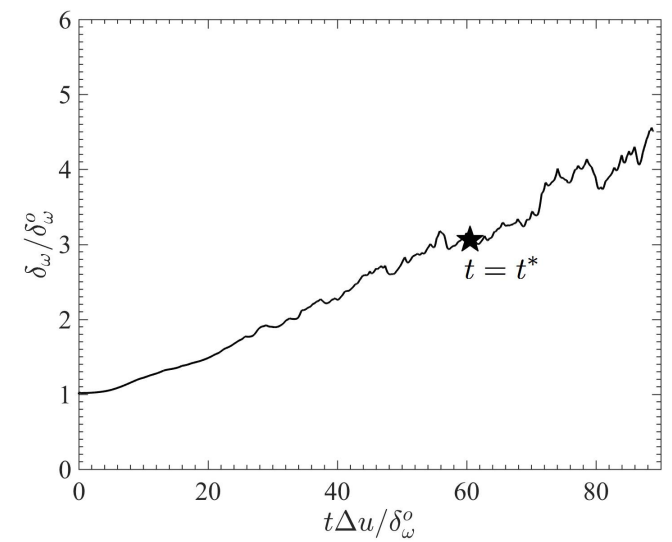

(a)

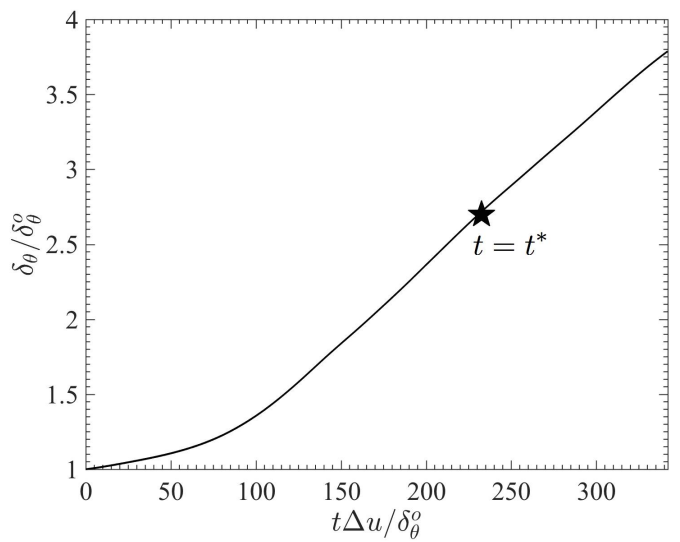

(b)

Figure 2. (a) Vorticity thickness as given by Eq. (1), and (b) momentum thickness Eq. (2) as a function of time. The star depicts the time at which ignition occurs $\left(t^{*}\right)$.

The three-dimensional compressible Navier-Stokes equations governing a multi-component chemically reacting mixture are discretized using narrow-stencil finite-difference operators that satisfy the summationby-parts (SBP) property. ${ }^{5}$ An explicit, sixth-order, centered finite difference is used in the domain interior, and third-order, one-sided finite differences are applied at the boundary. The domain is periodic in $x$ and $z$, and characteristic outflow conditions are applied at the domain extents in $y$ based on the simultaneousapproximation-term (SAT) ${ }^{16}$ boundary treatment, which when combined with the SBP operators provides a provable energy-stable scheme. To evaluate second and mixed derivatives, first derivative operators are applied consecutively, which necessitates the use of artificial dissipation to damp the highest wavenumber components supported by the grid. High-order accurate SBP dissipation operators ${ }^{17,18}$ are used that provide artificial viscosity based on a sixth-order derivative with a diffusion coefficient that depends on the local grid resolution. Time integration is by a fourth-order Runge-Kutta method with direct evaluation of the chemical source terms.

The chemistry is described by a single-step irreversible reaction,

$$
\text { Fuel }+s \text { Oxidizer } \rightarrow(1+s) \text { Products, }
$$

where $s$ is the mass stoichiometric ratio. The Damköhler number, $D a$, is chosen such that the characteristic laminar flame speed thickness $l_{F}=D / S_{L}$ is approximately $2 \Delta x$, where $D$ is the mass diffusivity and $S_{L}$ is the unstrained laminar flame speed for the stoichiometric mixture. The flame speed can be estimated for the simplified chemistry employed in this work using asymptotic and analytic models for lean flames, ${ }^{19}$

$$
S_{L} \approx \frac{2}{\beta} e^{-\frac{\beta}{2 \alpha}} \sqrt{\frac{M D a Y_{O \infty}}{R e_{\delta_{\omega}^{o}} \operatorname{Pr} \beta}},
$$

where $\beta$ is the Zel'dovich factor, $\alpha$ is the heat release parameter, $\operatorname{Pr}=0.7$ is the Prandtl number, and $M=0.6 \approx 2 M_{c}$ is the effective Mach number. The relevant parameters for the chemistry are taken to be $s=8, \beta=6$, and $\alpha=0.86$, which are representative of hydrogen-air mixtures. The values of $\alpha$ and $\beta$ were chosen to yield a flame temperature corresponding to that of hydrogen, while avoiding excessively stiff chemistry. The Damköhler number is set to $5 \times 10^{5}$, resulting in a laminar flame speed $S_{L}=\Delta u / 32$. 
The thermal effect of ignition is modeled as a source term in the transport equation for total (non chemical) energy, $\rho E$, by

$$
S_{\rho E}=P(t) \exp \left[-\frac{\left(x-x_{0}\right)^{2}}{2 r_{x}^{2}}-\frac{\left(y-y_{0}\right)^{2}}{2 r_{y}^{2}}-\frac{\left(z-z_{0}\right)^{2}}{2 r_{z}^{2}}\right] \text { with } P(t)=a \exp \left[-\frac{\left(t-t_{0}\right)^{2}}{2 \tau_{i g}^{2}}\right],
$$

where $\mathbf{x}_{0}=\left(x_{0}, y_{0}, z_{0}\right)$ is its center location of the thermal source peaking at $t=t_{0}$, with $r_{x}, r_{y}$, and $r_{z}$ setting the size of the source. The parameter $a$ sets the total energy input, and $\tau_{i g}$ sets its duration. As reported by Ballal and Lefebvre ${ }^{20}$ and employed by Mastoakos and co-workers, ${ }^{21,22}$ optimal spark duration varies between 0.2 to $0.4 \tau_{c}$, where $\tau_{c}=l_{F} / S_{L}$ is the characteristic chemical time scale. In this study, the ignition duration is $\tau_{i g}=0.2 \tau_{c}$. The radius is chosen such that it is smaller than the mixing layer thickness

and properly resolved by the grid. A value of $r_{x}=r_{y}=r_{z}=0.2 \delta_{\omega}^{o}$, corresponding to $10 \Delta x$ is used throughout.

\section{Ignition sensitivity}

\section{A. Formulation}

We wish to assess sensitivity of ignition to the set of parameters

$$
\theta=\left[\begin{array}{lllllllll}
a & x_{0} & y_{0} & z_{0} & r_{x} & r_{y} & z_{0} & t_{0} & \tau_{i g}
\end{array}\right]^{\top}
$$

in (5). Specifically, we wish to compute the gradient of a quantity of interest, $\mathcal{J}$, with respect to $\theta$, i.e., $\partial \mathcal{J} / \partial \theta_{i}$ for $i=1, \ldots, 9$ parameters. To quantify ignition success, a burning index $\mathcal{I}$ is defined as the integrated reduced temperature

$$
\mathcal{I}=\int_{\Omega} r(t)\left[\frac{T(\mathbf{x}, t)-T_{\infty}}{T_{f}-T_{\infty}}\right] d \mathbf{x},
$$

where $\Omega$ is the region of interest, $T$ is the temperature, $T_{f}$ is the adiabatic flame temperature, and $T_{\infty}$ is the free stream temperature of the air. Owing to the binary outcome of ignition, the sensitivity gradient may become ill-defined at the ignition boundary, which is problematic when seeking gradients in $\mathcal{J}$ from the corresponding adjoint solution. As described previously, ${ }^{14}$ variations in the burning index are relatively small immediately proceeding ignition, and thus integrating $\mathcal{I}$ within this short window yields a finite gradient. To this end, $r(t)$ is introduced such that $\mathcal{J}$ is sampled in a prescribed window that highlights the early ignition behavior. ${ }^{14}$

The quantity of interest is defined as

$$
\mathcal{J}(\mathbf{Q} ; \theta)=\int_{t_{1}}^{t_{2}} \mathcal{I}(\mathbf{Q} ; \theta) d t
$$

where $\mathbf{Q}=\left[\rho, \rho u_{i}, \rho E, \rho Y_{k}\right]^{\top}$ is the vector of conserved variables, which includes density $\rho$, momentum $\rho u_{i}$, total non-chemical energy $\rho E$, and $Y_{k}$ the density of species $k$, and $t_{1}$ and $t_{2}$ are the start and end times of the simulation. In general, $\mathcal{J}$ depends upon the flow solution $\mathbf{Q}$ and upon the set of modeling parameters $\theta$, and thus its variation is given by

$$
\delta \mathcal{J}=\left(\frac{\partial \mathcal{J}}{\partial \mathbf{Q}}\right)_{\theta} \delta \mathbf{Q}+\left(\frac{\partial \mathcal{J}}{\partial \theta}\right)_{\mathbf{Q}} \delta \theta
$$

In a multi-dimensional time-dependent flow, a direct evaluation of (9) is intractable, primarily due to the cost of computing the first term on the right-hand side involving $\delta \mathbf{Q}$. To this end, the adjoint equations are formulated in order to seek the functional dependence of $\mathcal{J}$ on the set of parameters $\theta$ without requiring repeated simulations. This is accomplished by introducing the adjoint variable $\mathbf{Q}^{\dagger}$ as a Lagrange multiplier to enforce adherence to the compressible Navier-Stokes equations $\mathcal{N}$ as a constraint,

$$
\mathcal{L}=\mathcal{J}-\int_{t_{1}}^{t_{2}} \int_{\Omega} \mathbf{Q}^{\dagger} \mathcal{N} d \mathbf{x} d t
$$


Note that the integrand is defined in the same manner as the cost functional for convenience in the adjoint formulation. Linearizing the Lagrangian with respect to the flow solution $\mathbf{Q}$ yields

$$
\delta \mathcal{L}=\delta \mathcal{J}-\int_{t_{1}}^{t_{2}} \int_{\Omega} \mathbf{Q}^{\dagger} \delta \mathcal{N} d \mathbf{x} d t
$$

The adjoint solution can be chosen to eliminate the dependence of $\delta \mathcal{J}$ on $\delta \mathbf{Q}$ through

$$
\frac{\delta \mathcal{J}}{\delta \theta}=\int_{t_{1}}^{t_{2}} \int_{\Omega} \mathbf{Q}^{\dagger} \frac{\partial S_{\rho E}}{\partial \theta} d \mathbf{x} d t
$$

As shown above, the sensitivity gradient is directly proportional to $\mathbf{Q}^{\dagger}$, and thus the accuracy of the numerical solution to the adjoint equation is of key importance. Without special care, discretization of the adjoint equations can degrade the accuracy of the resulting sensitivity gradients for unsteady or chaotic flows, ${ }^{23}$ which can become significant in the context of turbulence. A discrete-exact formulation yields sensitivity gradients that are machine-precision limited and not tied to the order of accuracy of the underlying numerical scheme. ${ }^{23,24}$ To this end, a space-time discrete-adjoint method recently developed for high-fidelity compressible turbulence simulations ${ }^{24}$ was recently extended in our previous work to chemically-reacting mixtures. ${ }^{14}$ In this work, the adjoint method is applied to the non-premixed hydrogen-air mixing layer shown in Fig. 1 to assess $\partial \mathcal{J} / \partial \theta_{i}$.

\section{B. Sensitivity of ignition placement}

The simulations were run until the initial transient was complete and $\delta_{\omega}$ and $\delta_{\theta}$ exhibited quasi-linear growth at time $t^{*}$, as depicted in Fig. 2, resulting in an effective Reynolds number of $R e_{\delta^{*}}=7000$. At this time the thermal source $S_{\rho E}$ was applied with $t_{0}=t^{*}+10 \tau_{i g}$ such that $P\left(t=t^{*}\right) \approx 10^{-14}$. A series of simulations were then conducted for a range of $\mathbf{x}_{0}$ to assess the influence of the local flow state on parametric sensitivity of ignition. The thermal source was first placed on the stoichiometric surface in the center of the domain with enough energy to ensure successful ignition, corresponding to $x_{0}=15.5 \delta_{\omega}^{o}, y_{0}=1.6 \delta_{\omega}^{o}, z_{0}=0$, and $a=3$. These parameters are used as a base case for comparison.

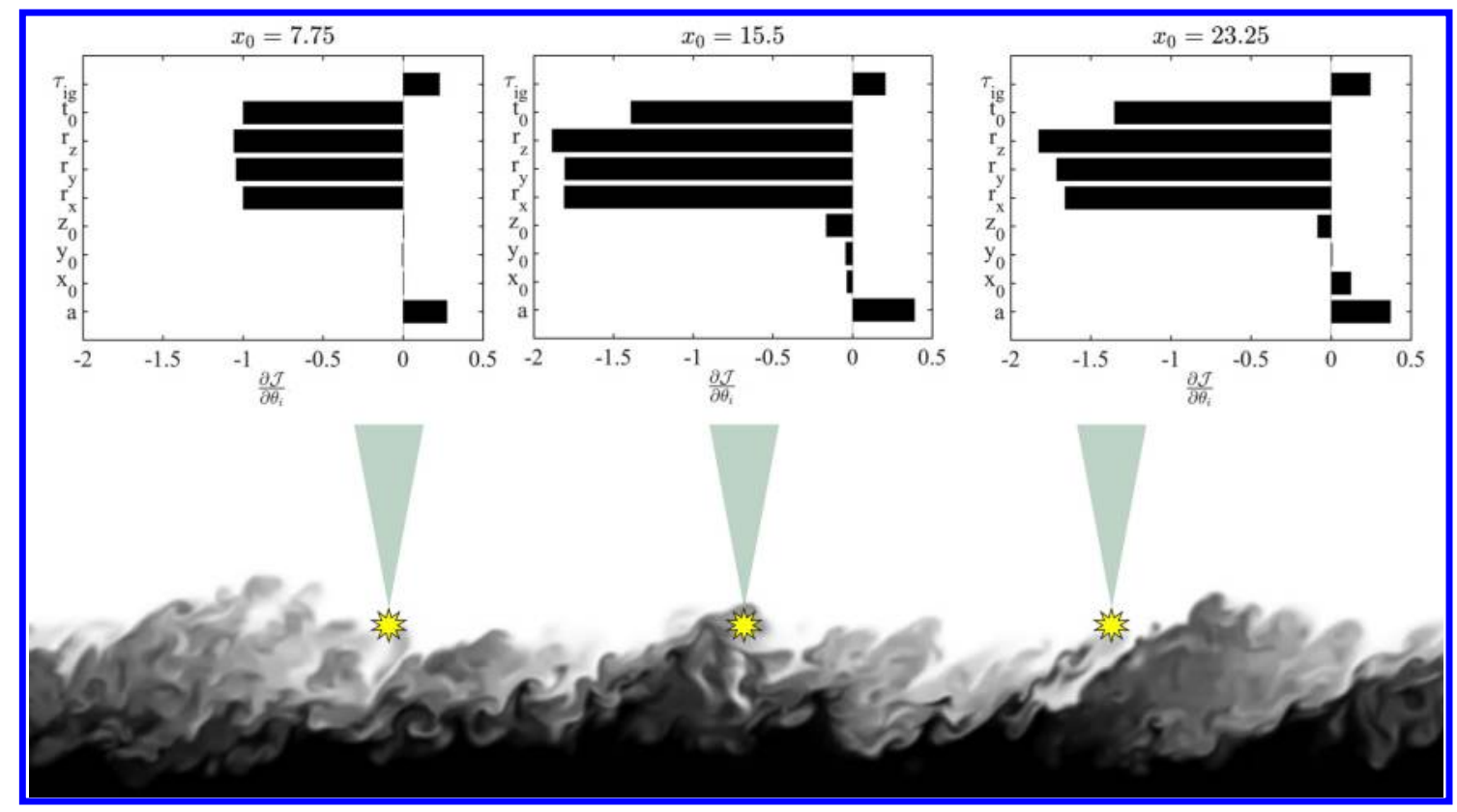

Figure 3. Parametric sensitivity at three horizontal locations in the mixing layer.

To assess the influence of the local flow properties on ignition, the thermal source was then placed at $x=7.75 \delta_{\omega}^{o}$ and $x=23.25 \delta_{\omega}^{o}$ (see Fig. 3), with all other parameters remaining the same. Note that because the flow is statistically homogeneous in $x$, varying the position of the igniter in this direction is equivalent 
to applying the ignition source at different flow realizations. Parametric sensitivity for each term appearing in the thermal source is shown in Fig. 3. It can be seen that for each case, the total energy amount $a$ is positive (i.e., increasing energy suggests a higher probability of ignition). In addition, the magnitude of the sensitivity of $\mathcal{J}$ to $a$ remains relatively constant at each position. The same can be seen for the ignition duration $\tau_{i g}$. At all positions, it is found that decreasing the ignition time $t_{0}$ and the radii $r_{x}, r_{y}$, and $r_{z}$, lead to positive sensitivity. Decreasing the spatial extent of the ignition source while keeping the total deposited energy unchanged would result in higher energy deposited over a smaller region. Thus, the adjoint solution suggests that depositing more energy in a smaller volume will lead to increased $\mathcal{J}$, and thus higher likelihood of ignition. The sensitivity of ignition placement $\mathbf{x}_{0}$ is seen to vary sign and magnitude at each location. This is likely due to local flow inhomogeneities caused by the turbulence resulting in varying strain rate and mixture composition at each position. It is interesting to find, however, that the majority of the parameters exhibit similar sensitivity at each location.

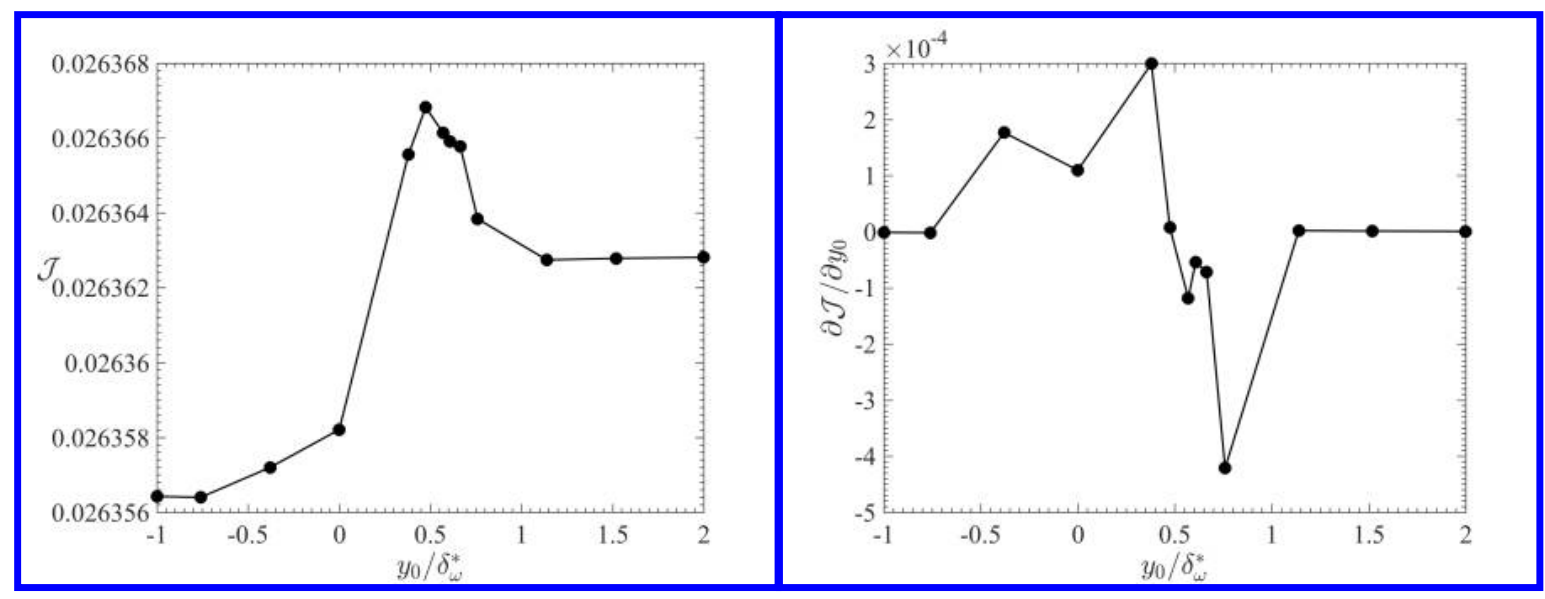

(a)

(b)

Figure 4. (a) Cost functional Eq. (8) and (b) its sensitivity to $y$ placement.

An additional set of simulations were conducted to assess the sensitivity of ignition to vertical placement in the shear layer. The composition changes from fuel and no oxidizer at $y \ll-\delta_{\omega}^{*}$ to pure air with no fuel at $y \gg \delta_{\omega}^{*}$. As shown in Fig. 4(a), the cost functional is greatest at $y_{0} \approx \delta_{\omega}^{*} / 2$. The cost functional is seen to remain constant for the cases with the thermal source located away from the mixing layer at $y_{0} \lesssim-\delta_{\omega}^{*}$ and $y_{0} \gtrsim-\delta_{\omega}^{*}$. The corresponding sensitivity to vertical placement is shown in Fig. 4(b). Away from the mixing layer the sensitivity is zero, and thus adjusting $y_{0}$ in these regions would have no effect on ignition likelihood. In general, positive sensitivity is observed for $y_{0}$ within the mixing layer below the position resulting in maximum $\mathcal{J}$, and negative sensitivity is observed for $y_{0}$ within the mixing layer above this optimal location.

\section{Conclusions}

The present studies presents direct numerical simulations of a turbulent non-premixed hydrogen-air shear layer subject to an external thermal source. A discrete adjoint method was used to measure sensitivity of the parameters used in modeling the thermal source on ignition. It was found that the majority of the parameters exhibit similar sensitivity regardless of the flow realization. This suggests that local sensitivity obtained from an adjoint method can provide consistent information in unsteady flows. Parametric uncertainty was also computed for various vertical placements of the igniter. It was found that placing the ignition source outside the vorticity thickness results in no sensitivity to the vertical placement. Inside the vorticity thickness, the adjoint solution was found to provide a direction to adjust the parameters to maximize the cost functional. While these results show promise for eventually using adjoint-based methods for optimizing design of turbulent combustion systems, it remains to be seen how well the sensitivity gradients behave for longer time horizons. For the ignition studies considered herein, the cost functional was only needed to be measured over a short time horizon. Thus, any cumulative error growth in the adjoint gradient that is known 
to arise from chaos was not present.

\section{Acknowledgment}

This material is based in part upon work supported by the Department of Energy, National Nuclear Security Administration, under Award Number DE-NA0002374. This research used resources of the Oak Ridge Leadership Computing Facility, which is a DOE Office of Science User Facility supported under Contract DE-AC05-00OR22725. This research also used resources of the Argonne Leadership Computing Facility, which is a DOE Office of Science User Facility supported under Contract DE-AC02-06CH11357. 


\section{References}

${ }^{1}$ Korkegi, R. H., "Comparison of shock-induced two-and three-dimensional incipient turbulent seperation," AIAA Journal, Vol. 13, No. 4, 1975, pp. 534-535.

${ }^{2}$ Korkegi, R. H., "A lower bound for three-dimensional turbulent separation in supersonic flow," AIAA Journal, Vol. 23, No. 3, 1985, pp. 475-476.

${ }^{3}$ Brieschenk, S., Kleine, H., and O'Byrne, S., "Laser ignition of hypersonic air-hydrogen flow," Shock Waves, Vol. 23, No. 5, 2013, pp. 439-452.

${ }^{4}$ Ballal, D. and Lefebvre, A., "The influence of spark discharge characteristics on minimum ignition energy in flowing gases," Combustion and Flame, Vol. 24, 1975, pp. 99-108.

-5trand, B., "Summation by parts for finite difference approximations for d/dx," Journal of Computational Physics, Vol. 110, No. 1, 1994, pp. 47-67.

${ }^{6}$ Daou, J. and Daou, R., "Flame balls in mixing layers," Combustion and Flame, Vol. 161, No. 8, 2014, pp. 2015-2024.

${ }^{7}$ Drummond, J. P., Diskin, G. S., and Cutler, A. D., "Fuel-air mixing and combustion in scramjets," 2002.

${ }^{8}$ Mastorakos, E., "Ignition of turbulent non-premixed flames," Progress in Energy and Combustion Science, Vol. 35, No. 1, 2009, pp. 57-97.

${ }^{9}$ Ahmed, S. F. and Mastorakos, E., "Spark ignition of lifted turbulent jet flames," Combustion and Flame, Vol. 146, No. 1, 2006, pp. 215-231.

${ }^{10}$ Ahmed, S. F., Balachandran, R., and Mastorakos, E., "Measurements of ignition probability in turbulent non-premixed counterflow flames," Proceedings of the Combustion Institute, Vol. 31, No. 1, 2007, pp. 1507-1513.

${ }^{11}$ Lacaze, G., Richardson, E., and Poinsot, T., "Large eddy simulation of spark ignition in a turbulent methane jet," Combustion and Flame, Vol. 156, No. 10, 2009, pp. 1993-2009.

$\checkmark{ }^{12}$ Bane, S., Ziegler, J., Boettcher, P., Coronel, S., and Shepherd, J., "Experimental investigation of spark ignition energy in kerosene, hexane, and hydrogen," Journal of Loss Prevention in the Process Industries, Vol. 26, No. 2, 2013, pp. 290-294.

${ }^{13}$ Eyssartier, A., Cuenot, B., Gicquel, L., and Poinsot, T., "Using LES to predict ignition sequences and ignition probability of turbulent two-phase flames," Combustion and Flame, Vol. 160, No. 7, 2013, pp. 1191-1207.

${ }^{14}$ Capecelatro, J., Vishnampet, R., Bodony, D. J., and Freund, J. B., "Adjoint-based sensitivity analysis of localized ignition in a non-premixed hydrogen-air mixing layer," 54th AIAA Aerospace Sciences Meeting, 2016, p. 2153.

${ }^{15}$ Pantano, C., Sarkar, S., and Williams, F., "Mixing of a conserved scalar in a turbulent reacting shear layer," Journal of Fluid Mechanics, Vol. 481, 2003, pp. 291-328.

$\checkmark 16$ Bodony, D. J., "Accuracy of the simultaneous-approximation-term boundary condition for time-dependent problems," Journal of Scientific Computing, Vol. 43, No. 1, 2010, pp. 118-133.

$\checkmark{ }^{17}$ Mattsson, K., Svärd, M., and Nordström, J., "Stable and accurate artificial dissipation," Journal of Scientific Computing, Vol. 21, No. 1, 2004, pp. 57-79.

${ }^{18}$ Vishnampet Ganapathi Subramanian, R., An exact and consistent adjoint method for high-fidelity discretization of the compressible flow equations, Ph.D. thesis, University of Illinois at Urbana-Champaign, 2015.

${ }^{19}$ Poinsot, T. and Veynante, D., Theoretical and numerical combustion, Edwards, 2005.

${ }^{20}$ Ballal, D. R. and Lefebvre, A., "Spark ignition of turbulent flowing gases," Proceedings of the 15th Aerospace Sciences Meeting, AIAA Los Angeles, Paper, No. 77-185, 1977.

${ }^{21}$ Chakraborty, N. and Mastorakos, E., "Numerical investigation of edge flame propagation characteristics in turbulent mixing layers," Phys. of Fluids, Vol. 18, No. 10, 2006, pp. 105103.

${ }^{22}$ Chakraborty, N. and Mastorakos, E., "Direct numerical simulations of localised forced ignition in turbulent mixing layers: the effects of mixture fraction and its gradient," Flow, Turbulence and Combustion, Vol. 80, No. 2, 2008, pp. 155-186.

${ }^{23}$ Nadarajah, S. K. and Jameson, A., "A comparison of the continuous and discrete adjoint approach to automatic aerodynamic optimization," Proceedings of the 38th Aerospace Sciences Meeting and Exhibit, AIAA Reno, NV, Paper, 2000.

${ }^{24}$ Vishnampet, R., Bodony, D. J., and Freund, J. B., "A practical discrete-adjoint method for high-fidelity compressible turbulence simulations," J. Comp. Phys., Vol. 285, 2015, pp. 173-192. 